\title{
HIV Rapid Testing in a VA Primary Care Department Setting: Programmatic Cost Analysis at Five Years
}

\section{Herschel Knapp ${ }^{1 *}$ and Kee Chan ${ }^{2}$}

${ }^{1}$ Veterans Affairs (VA) Quality Enhancement Research Initiative for HIV and Hepatitis (QUERI-HIV/HEP) and Center for the Study of Healthcare Provider Behavior, VA Greater Los Angeles Health Services Research and Development (HSR\&D) Center of Excellence, VA Greater Los Angeles Healthcare System, Los Angeles, CA, USA ${ }^{2}$ Department of Health Sciences, Sargent College of Health and Rehabilitation Sciences, Boston University, USA

\begin{abstract}
Patients with HIV benefit from early diagnosis, however, the approach and cost of HIV testing is often unknown. Thus, this study explored the implementation of a universal (non-risk-based) nurse-initiated HIV rapid testing program in the Primary Care Clinic at the Veterans Health Administration, Los Angeles Ambulatory Care Clinic near Downtown Los Angeles. Over the course of the first five years of this program, nurses administered a total of 2,117 tests. The mean programmatic cost of the first five years is estimated at about $\$ 4,868$ (SD $\$ 1,344)$ per year ( $\$ 11.50$ per test). The overall cost of the implementation may have slightly increased cost but within a reasonable range.
\end{abstract}

Keywords: HIV; Rapid testing; Diagnostic; Infectious disease; Screening

\section{Background}

Among the approximately 1.2 million persons living with HIV in the United States, it is estimated that as many as $1 / 3$ of these individuals are unaware of their status [1]. The consequences associated with these (asymptomatic) unidentified individuals are twofold: (1) They do not receive the necessary treatment in a timely fashion, and (2) they may be unknowingly transmitting the disease to others [2]. Effective antiretroviral therapy has reformed the outlook of HIV from a nonnegotiable death sentence to a manageable chronic disease [2]. Since the development and use of HAART (highly active antiretroviral therapy), HIV mortality rates are largely associated with late diagnosis, leading to a delay in treatment [3]. In an effort to increase HIV detection, diagnostic guidelines have been revised to recommend a shift from risk-based testing, to universal testing of all patients at least once per lifetime, starting at age 13 [4]. The U.S. Department of Veterans Affairs (VA), the largest single provider of HIV care in the country), has recently adopted this recommendation [5].

Current testing methods have been shown to be highly ineffective, due largely to the method itself. Conventional HIV testing requires both a blood draw and laboratory analysis, requiring a patient to return days or even weeks later to receive test results. Consequently, a significant number of people simply do not return for their results. This is vitally important, for research shows that when people are aware of their status, they tend to cease or lessen their risk-related behaviors, which in turn works to reduce further spread of the epidemic [1]. Lack of return for test results has been shown to be a significant contributor to the HIV epidemic. In a recent study, almost $1 / 3$ of those who tested HIVpositive did not return for results. This unawareness among (potentially asymptomatic) infected individuals has adverse implications for the spread of the HIV epidemic; CDC data suggest that such individuals are 3.5 times more likely to infect others than those who are aware of their HIV status [4].

An additional barrier is needle phobia; the formal diagnosis is "Specific Phobia, Blood-injection-injury type", which has a $10 \%$ prevalence among children and adults. The symptoms of this phobia go beyond ordinary needlestick anxiety [6]; as such, these patients tend to resist seeking medical care, placing them at greater risk for a variety of undiagnosed diseases [7]. To address these barriers, nurse-initiated HIV oral rapid testing (HIV RT), which produces results while-you-wait, has been proposed as a viable remedy to increase screening in general medical and urgent care settings [1]. The FDA approved OraQuick Advance $^{\mathrm{TM}}$ rapid test, which produces reliable ELISA (enzyme-linked immunosorbent assay) results in 20 minutes based on a noninvasive sample of oral fluid, alleviates the need for venipuncture and the need for patients to return for results.

Another advantage of the HIV RT is that patients are more amenable to such testing in that they need no longer endure weeks of anxiety waiting for test results [8]. In a previous randomized, controlled trial within the VA, nurse-initiated HIV rapid testing (NRT) was found to be more acceptable to patients than current venipuncture methods and also significantly increased receipt of test results [9].

Nurse-initiated screening (sometimes called "standing orders") systematizes testing into primary prevention priorities. Use of the HIV RT has been widely applied in nonprimary care settings, and is acceptable to both patients and providers.

\section{Objective}

The objective of this manuscript is to evaluate the cost of nurse-initiated HIV RT in a VA primary care clinic in Los Angeles, California [9].

\section{Methods}

\section{Study site}

This implementation took place at the Veterans Health

*Corresponding author: Herschel Knapp, Veterans Affairs (VA) Quality Enhancement Research Initiative for HIV and Hepatitis (QUERI-HIV/HEP) and Center for the Study of Healthcare Provider Behavior, VA Greater Los Angeles Health Services Research and Development (HSR\&D) Center of Excellence, VA Greater Los Angeles Healthcare System, Los Angeles, CA, USA, Tel: (310) 4783711; E-mail: Herschel.Knapp@va.gov

Received February 11, 2015; Accepted April 17, 2015; Published April 22, 2015

Citation: Knapp H, Chan K (2015) HIV Rapid Testing in a VA Primary Care Department Setting: Programmatic Cost Analysis at Five Years. J AIDS Clin Res 6 : 451. doi:10.4172/2155-6113.1000451

Copyright: (C) $2015 \mathrm{Knapp} \mathrm{H}$, et al. This is an open-access article distributed under the terms of the Creative Commons Attribution License, which permits unrestricted use, distribution, and reproduction in any medium, provided the original author and source are credited. 
Administration Los Angeles Ambulatory Care Clinic, a comprehensive care outpatient clinic situated near downtown Los Angeles. Our specific target was the Primary Care Clinic at that facility.

\section{Patient selection criteria}

Per VHA guidelines, nurses offered HIV RT screening to patients universally (as opposed to risk-based criteria). Only those who had a prior diagnosis of HIV or AIDS were appropriately excluded.

\section{Training}

Nurses at the Veterans Los Angeles Ambulatory Primary Care Clinic who were interested in becoming certified in administering the HIV RT were required to attend two 90 minute in-services: Part 1 was conducted by a clinical social worker and entailed HIV education, therapeutic communication skills, techniques for effectively proposing HIV testing, pretest counseling, completing the informed consent documentation (which was mandatory at that time), a demonstration of the test instrument detailing test storage, test administration and interpretation, test disposal, entering the test outcome into the VA electronic medical record, and posttest counseling. Part 2 was administered by the laboratory manager; this hands-on tutorial trained practitioners how to conduct the manufacturer's specified quality control procedures. The training concluded with nurses demonstrating competency in properly interpreting simulated test outcomes for negative and (preliminary) positive results.

\section{Consent}

During the first 17 months of this implementation (April, 2008August, 2009), written informed consent was required prior to conducting an HIV test; this involved a three page document paired with about five minutes of pretest counseling. After August, 2009, the policy shifted to verbal consent for HIV testing, which involved about one minute, and no special paperwork or patient signature.

\section{Testing}

As part of the usual check-in process wherein vital signs were gathered, nurses routinely offered patients the HIV RT, after which, they would return to the waiting area. Nurses imparted negative results to patients in a confidential manner. In cases where the HIV RT produced (preliminary) positive results, the nurse would notify the primary care physician, who would then disclose the results to the patient. We configured our electronic medical record system to automatically generate the following orders for (preliminary) positive HIV RTs: Blood draws for (confirmatory) Western Blot, viral load, CD4 cell count, and a referral to the Infectious Disease specialist to facilitate optimal care.

\section{Results}

During this six hour launch event, the team of nurses administered 83 HIV RTs. Over the course of the next five years (April 1, 2008 through March 31, 2013), nurses at this site administered a total of 2,117 HIV RTs; this works out to a quarterly mean of $106(\mathrm{SD}=50.6)$ HIV RTs. Despite the variability of the testing rate, the positive slope of the (dotted) trendline depicts the average quarterly increase of 2.6 additional tests per quarter over this timeframe (Figure 1).

The prelaunch planning meetings involved the research implementation lead, facility nurse manager, laboratory lead, nurses, and patient education professionals at the targeted facility. The research implementation team that initiated this project was stationed at the Greater Los Angeles VA campus, 15 miles from the Downtown Los Angeles VA Clinic. After the initial launch, the members of the research implementation team did not maintain regular contact with that site. The staff at the targeted clinic took active ownership of the HIV RT process resulting in a self-sustaining implementation [10].

\section{Cost analysis}

We analyzed the budget associated with the first five years of this HIV RT implementation spanning April, 2008 through March, 2013. We assessed the cost of the HIV RT test kit at $\$ 10.09$ and the personnel cost associated with pretest counseling (Table 1). Quarters 1-4 represents the timeframe wherein pretest counseling along with the mandatory written informed consent was required. This process involved the nurse reviewing the three-page informed consent document and having (willing) patients sign it prior to administering the HIV test; this process took about five minutes. After the fourth quarter, the pretest protocol changed from the three page written consent document that required

\section{HIV Oral Rapid Testing in Primary Care Clinic}

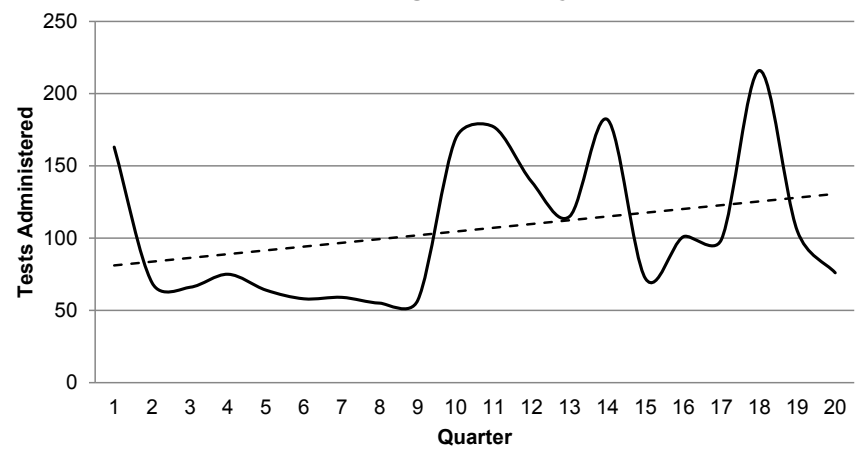

Note: Dotted (regression) trendline represents 2.6 additional tests per quarter Figure 1: Quarterly HIV RT counts: April, 2008-March, 2013.

\begin{tabular}{|c|c|c|c|c|c|c|}
\hline Quarter & $1-4$ & $5-8$ & $9-12$ & $13-15$ & $16-20$ & Total \\
\hline Number of test+ & 373 & 236 & 542 & 470 & 496 & 2,117 \\
\hline HIV testing cost+ & $\$ 3,763.57$ & $\$ 2,381.24$ & $\$ 5,468.78$ & $\$ 4,642.30$ & $\$ 5,004.64$ & $\$ 21,260.53$ \\
\hline Personnel cost time\# & $\$ 1,398.75$ & $\$ 177.00$ & $\$ 406.50$ & $\$ 352.50$ & $\$ 372.00$ & $\$ 2,706.75$ \\
\hline Implementation program cost & $\$ 5,162.32$ & $\$ 2,558.24$ & $\$ 5,875.28$ & $\$ 4,994.80$ & $\$ 5,748.64$ & $\$ 24,339.28$ \\
\hline Average cost per test & $\$ 13.84$ & $\$ 10.84$ & $\$ 10.84$ & $\$ 10.62$ & $\$ 11.59$ & $\$ 11.50$ \\
\hline
\end{tabular}

*Quarters 1-4 involved the five minute pretesting counseling and written informed consent with patient signature required before the August, 2009. After August, 2009, the consent process shifted to verbal consent for HIV testing which required about one minute.

+Federal pricing for HIV RT kit for Veteran Health Affairs is \$10.09.

\#On average, pretesting counseling required about five minutes; based on average nurse salary of $\$ 45.05$ per hour at this facility, we estimated $\$ 3.75$ for the five minute consent process, and $\$ 0.75$ for one minute verbal consent process.

Table 1: Quarterly cost analysis of HIV testing program. 
the patient's signature to verbal consent, which required only about one minute in Quarters 5-20. Quarters 5-8 had a low upfront cost of the implementation of $\$ 2,558$. The cost of the implementation in Quarters 1-4 and Quarters 5-20 are similar which suggests that even with the increased number of HIV test performed, the reduction of time (about one minute) associated with verbal (not written and signed) pretest counseling reduced costs.

\section{Conclusion}

Based on the findings of this study, the cost of implementation expectedly varies across quarters. While there are upfront costs to the implementation of HIV testing, the overall benefits of the increased number of HIV tests performed outweigh the cost. The cost of the program decreased in Quarters 5-8, which may imply an initial saturation of HIV tests performed. However, in Quarters 9-12, the cost of implementation rose to $\$ 5,800$, which is close to the beginning of the implementation (Quarters 1-4). This may represent the cyclic pattern of the programmatic cost that healthcare managers should anticipate in terms of budget allocation in reproducing this implementation.

Due to nursing staffing shortage in the year(s) prior to our implementation, it is considered that pre- or post-analyses of HIV testing rate would artificially inflate the effectiveness of our intervention. In future research, we intend to capture this data to determine our rates of HIV testing and positives detected among patients screened using RT HIV universal testing. Our current feasibility study provided us with an essential set of key parameters, such as salary of health professional, time-motion data, and cost of test, to pursue a follow-up cost analysis.

The findings from our study highlight the potential benefits from the cost-savings at individual and macro level. When HIV is detected early, patients benefit from more affordable treatment with potentially better outcomes. Although we were not able to collect the hospitalization costs related to HIV care from the patients in our program, we believe that early detection of these patients could result in early treatment and effective management of care that may result in avoidable hospitalization(s). Future research will include a prospective analysis of the cost involved in the pre- and post-program care such as the inpatient and outpatient visit pertaining to HIV identification. In most cases, patients with late stage or late detection may have a poorer outlook, requiring costly ED visits, and possibly hospitalizations, depending on the acuity. At a macro level, individuals who know they are HIV positive tend to reduce their unprotected encounters, thereby reducing the costs associated with treating additional HIV positive patients. The lifetime cost for a HIV patient with $\leq 200 \mathrm{CD} 4 \mathrm{~T}$ cells $/ \mu \mathrm{l}$ is $\$ 253,000$ compared to patients with $501-900 \mathrm{CD}$ T cells $/ \mu \mathrm{l}$ is $\$ 402,000$ [11]. Early diagnosis and treatment of HIV infection increases lifetime costs but additional life years from 30.8 to 38.1 for patients with late and early detection, respectively [11].

While there are may be some upfront cost related to the testing and care of HIV patients, the long-term savings of avoidable hospitalization(s) associated with this chronic condition will outweigh the implications of the short-term cost.

\section{References}

1. Centers for Disease Control and Prevention (CDC) (2011) HIV surveillance-United States, 1981-2008. MMWR Morb Mortal Wkly Rep 60: 689-693.

2. Palella FJ Jr, Deloria-Knoll M, Chmiel JS, Moorman AC, Wood KC, et al. (2003 Survival benefit of initiating antiretroviral therapy in HIV-infected persons in different CD4 cell strata. Annals of Intern Med 138: 620-626.

3. Anastos K, Barrón Y, Miotti P, Weiser B, Young M, et al. (2002) Risk of progression to AIDS and death in women infected with HIV-1 initiating highly active antiretroviral treatment at different stages of disease. Arch Intern Med 162: 1973-1980.

4. Branson BM, Handsfield HH, Lampe MA, Janssen RS, Taylor AW, et al. (2006) Revised recommendations for HIV testing of adults, adolescents, and pregnant women in health-care settings. MMWR Recomm Rep 55: 1-17.

5. Wagner TH, Taylor T, Cowgill E, Asch SM, Su P, et al. (2015) Intended and unintended effects of large-scale adverse event disclosure: a controlled beforeafter analysis of five large-scale notifications. BMJ Qual Saf 24: 295-302.

6. American Psychiatric Association (2013) Diagnostic and Statistical Manual of Mental Disorders. DSM 5, Washington DC, APA, p. 197.

7. Hamilton JG (1995) Needle phobia: a neglected diagnosis. J Fam Pract 41 169-175.

8. Galvan FH, Brooks RA, Leibowitz AA (2004) Rapid HIV testing: issues in implementation. AIDS Patient Care STDS 18: 15-18.

9. Anaya HD, Bokhour B, Feld JE, Golden J, Asch SM, et al. (2012) Qualitative Implementation of Routine Rapid HIV Testing Within the US Department of Veterans Affairs Healthcare System. Journal for Healthcare Quality 34: 7-14.

10. Knapp H, Anaya HD, Goetz MB (2010) Attributes of an independently selfsustaining implementation: nurse-administered HIV rapid testing in VA primary care. Qual Manag Health Care 19: 292-297.

11. Farnham PG, Gopalappa C, Sansom SL, Hutchinson AB, Brooks JT, et al (2013) Updates of lifetime costs of care and quality-of-life estimates for HIVinfected persons in the United States: late versus early diagnosis and entry into care. J Acquir Immune Defic Syndr 64:183-189. 